\title{
Understanding Bacteriophage Therapy as a Density-dependent Kinetic Process
}

\author{
Robert J. H. Payne*† and Vincent A. A. Jansent@ \\ *Department of Zoology, University of Oxford, South Parks Road, Oxford OX1 3PS, U.K. and \\ $\ddagger W$ ellcome Trust Centre for the Epidemiology of Infectious Diseases, University of Oxford, \\ South Parks Road, Oxford OX1 3PS, U.K.
}

(Received on 14 December 1999, Accepted in revised form on 13 September 2000)

\begin{abstract}
Studies of bacteriophage as therapeutic agents have had mixed and unpredictable outcomes. We argue that interpretation of these apparently paradoxical results requires appreciation of various density-dependent threshold effects. We use a mathematical model to delineate different categories of outcome, including therapy by simple inundation, by active biocontrol, and by delayed active biocontrol. Counter-intuitively, there are situations in which earlier inoculation can be less efficacious, and simultaneous inoculation with antibiotics can be detrimental. Predictions of therapeutic responses are made using formulae dependent on biologically meaningful parameters; experimental measurement of the parameters will be a prerequisite of application of the model to particular study systems. Such modelling can point to which aspects of phage biology might most fruitfully be engineered so as to enhance the viability of bacteriophage therapy.
\end{abstract}

(C) 2001 Academic Press

\section{Introduction}

Given the apparently inexorable advent of the post-antibiotic era (Berkowitz, 1995; Tenover \& Hughes, 1996) it is surprising how few rigorous studies have focussed on the therapeutic alternative of bacteriophage biocontrol. Two recent reviews (Barrow \& Soothill, 1997; Alisky et al., 1998) summarize results from experimental work both on animals and on people. Although these reviews point clearly to the potential of phage

\footnotetext{
$\dagger$ Author to whom correspondence should be addressed. Current address: School of Animal and Microbial Sciences, University of Reading, Whiteknights, Reading RG6 6AJ, U.K. E-mail: robert.payne@zoo.ox.ac.uk

$\S$ Current address: School of Biological Sciences, Royal Holloway, University of London, Egham, Surrey TW20 OEX, U.K.
}

therapy as a weapon against antibiotic-resistant bacteria under certain circumstances, they also serve to highlight our ignorance of important areas of the phage-bacteria interaction.

Table 1 lists some typical results from experimental studies, illustrating the range of outcomes. All the phage listed demonstrated active replication in culture, and yet showed widely varying activity when used in living hosts. Although sometimes active phage replication is successfully found in vivo, more often than not there is no active replication, with therapeutic benefits only being obtained by using very large or repeated doses, or not at all.

This uncertainty of transfer from behaviour in culture to behaviour in live hosts has dogged studies of phage as putative therapeutic agents ever since the idea was first advocated during the 
TABLE 1

A selection of results of phage therapy research

\begin{tabular}{lll}
\hline Bacterial infection & Phage & Results (reference) \\
\hline Salmonella typhimurium & Salmonella phage & Need large dose [a] \\
Pseudomonas aeruginosa & Pseudomonas phage & Need large dose [b] \\
Acinetobacter baumannii & Acinetobacter phage & Only need low dose [b] \\
Staphylococcus aureus & Staphylococcus phage & No effect [b] \\
Escherichia coli & Anti-K1 phage & Only need low dose [c] and [d] \\
Dysentery & Coli-Proteus & Need several courses [e] \\
Dysentery & Anti-pseudomonas & Only need one course [e] \\
Staphylococcus sp. & Gram-negative & Use of antibiotic adjuvant \\
& & diminished efficacy from \\
& & 95.2 to 84.9\% [f]
\end{tabular}

References: [a] Berchieri et al. (1991), [b] Soothill (1992), [c] Smith \& Huggins (1982), [d] Smith et al. (1987a), [e] Tolkachera et al. (1981), [f] Slopek et al. (1983).

First World War. Although great enthusiasm greeted the discovery of bacteriophage (by Twort in 1915 and independently by d'Herelle in 1917), this enthusiasm gradually waned following failure of various poorly conceived studies during the 1920s and 1930s, and almost completely lapsed once the introduction of antibiotics apparently obviated the need for other approaches. Various arguments have been proffered for the failure of the early studies, including lack of awareness of phage-bacteria specificity, presence of contamination, horizontal transfer of toxins by temperate phage, action of host anti-phage immune responses, and emergence of phageresistant bacterial mutants.

In this paper, we suggest that many of the apparently enigmatic facets of phage behaviour are a simple consequence of kinetic properties of phage replication. We draw on an understanding of the principles of population dynamics to support this viewpoint, and to create a framework for a theory of the optimal treatment strategies in bacteriophage therapy. We construct a simple but generic mathematical model to capture the critical replication and densitydependent qualities of bacteria-bacteriophage interactions, based on a minimal set of the essential biological processes at work. The model allows formulae to be derived which predict therapy outcome dependent on the life-history parameters, the inoculum size, and the inoculum timing.

\section{A Kinetic Model of Phage Infection}

$$
\text { MODEL FORMULATION }
$$

Let $x(t)$ represent the concentration of uninfected bacteria, $y(t)$ the lytic bacteria, and $v(t)$ the free phage. The change in concentrations over time are described by the differential equations

$$
\begin{gathered}
\frac{\mathrm{d} x}{\mathrm{~d} t}=a x-b v x-H(t) x, \\
\frac{\mathrm{d} y}{\mathrm{~d} t}=a y+b v x-k y-H(t) y, \\
\frac{\mathrm{d} v}{\mathrm{~d} t}=k L y-b v x-m v-h(t) v
\end{gathered}
$$

Here $a$ is the replication coefficient of the bacteria, $b$ is the transmission coefficient, $k$ the lysis rate, $L$ the burst size, and $m$ the decay rate of free phage. We assume the replication rate of infected bacteria to be the same as for uninfected bacteria, but it would be easy to adapt the equations for situations where this assumption does not hold, by using a suitable redefinition of the parameter $k$. The initial input of bacteria is of size $x_{0}$ at time zero; inoculation of an amount $v_{\phi}$ of phage is given at time $t_{\phi}$.

Any host responses against the bacteria or against the phage can be incorporated via the variables $H(t)$ and $h(t)$, respectively (here, and 
throughout, we reserve the word "host" to refer to the host of the bacteria). There are many types of bacterial infections, including gastrointestinal infections, suppurative wound infections, antibiotic-associated dysbacteriosis, sepsis, and infections secondary to other conditions. Consequently, there are likewise many forms of natural host responses. In addition, there could be homeostatic immune mechanisms wherein the host responses to the bacteria affect the phage and vice versa. Rather than seeking the details of specific responses such as the effects of flushing (diarrhoea), or of the immune system, we use the variables $H(t)$ and $h(t)$ to capture the role of the host in a generic manner.

This model is appropriate for lytic phage but not for temperate phage, which do not lyse bacteria but instead enter a latent lysogenic state. Temperate phage are not used in phage therapy because they reproduce more slowly and often code for toxin production.

For simplicity, the model does not include the possibility of bacterial growth constrained by target cell limitation. In the context of phage therapy, target cell limitation could change the details of the outcome, but would not change the qualitative features that we report here.

Exact parameter values are not widely reported, but are likely to vary considerably from system to system. Approximate estimates are available from modelling studies (Levin \& Bull, 1996; Schrag \& Mittler, 1996), and by inference from time-series data in experimental studies (Smith \& Huggins, 1982; Merril et al., 1996). For computer simulations of the model we examined a range of values giving, where possible, biologically plausible rates of turnover, decay, lysis and burst size. The computer simulations demonstrate that even this "minimal" model is capable of exhibiting a range of qualitatively different outcomes, using only a handful of different initial conditions and different parameter values. Figure 1(a) shows how a sufficiently large dose of phage can eradicate the bacteria even without active replication; Fig. 1(b) shows how a slightly smaller initial dose might result in only a transient inundation effect; Fig. 1(c) shows how for different parameter values a small dose can be sufficient to lead to active replication of phage that subsequently fully subdues the bacterial
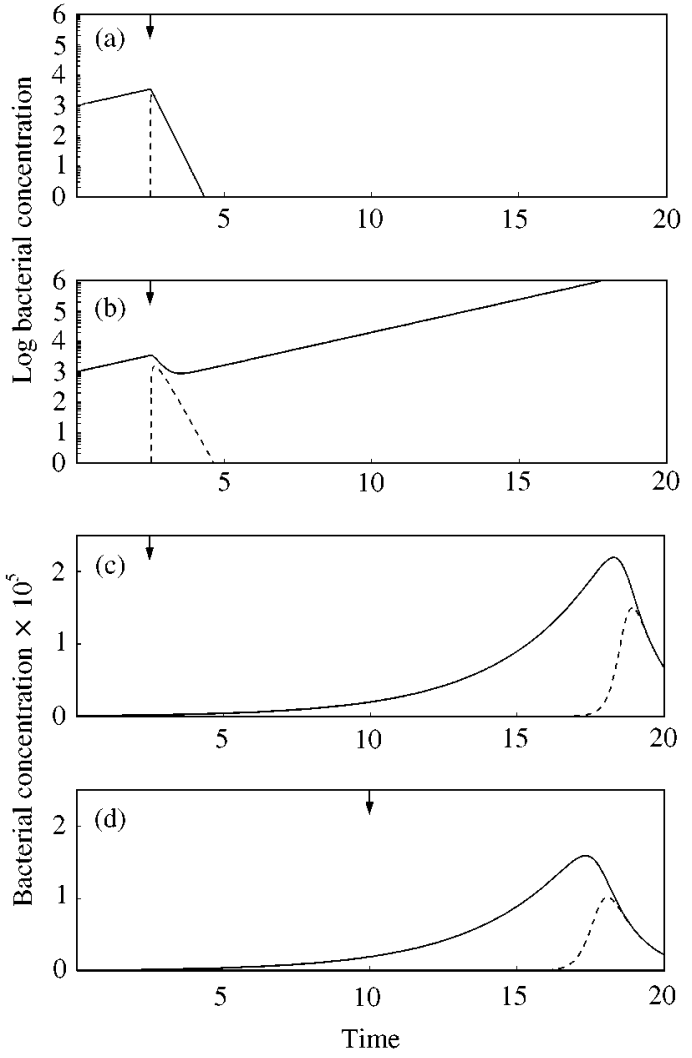

FIG. 1. Different qualitative outcomes of phage treatment. Times of inoculation are marked by arrows. In (a) and (b) relatively large doses of phage are administered. In (a) this results in almost immediate clearance of the bacterial infection; in (b) some reduction of the bacterial concentration is achieved without clearing the infection, allowing the bacterial population to regrow. In (c) a small dose of phage is given which results in control of the bacterial infection at a later point in time; in (d) delayed administration of phage also results in control of bacterial infection, but does so earlier and for a lower peak bacterial density. All graphs were computed from numerical solutions of eqns (1-3). The solid lines represent the total bacterial concentration $(x(t)+y(t))$, the dashed lines the fraction of phage-infected bacteria $[y(t)]$. In all simulations the initial dose of bacteria was $x_{0}=1000, y_{0}=0$. Time is measured in hours. (a) Parameters: $a=0.5, b=10^{-7}, k=5, L=100, m=5$; phage inoculation $t_{\phi}=2.5, v_{\phi}=10^{9}$. (b) As for (a) except $v_{\phi}=10^{8}$. (c) Parameters: $a=0.3, b=10^{-6}, k=1.2, L=100, m=1.8$; phage inoculation $t_{\phi}=2.5, v_{\phi}=100$. (d) As for (c) except $t_{\phi}=12.5$.

infection; comparison of Fig. 1(c) with Fig. 1(d) illustrates an interesting effect - that this form of active therapy can sometimes be more efficacious when the time of inoculation is delayed [Fig. 1(d) uses identical parameters and initial conditions as Fig. 1(c), except for timing of phage inoculation, as marked by arrows]. 
This diversity of results may seem bewildering, but in the next section we show how it is possible to analytically predict the circumstances under which these and other categories of outcome manifest.

\section{CRITICAL CONCENTRATIONS AND CRITICAL TIMES}

There is a threshold density of bacteria that must be present in order for the virus numbers to increase, which we shall refer to as the proliferation threshold, $X_{P}$ (this is analogous to the eradication threshold used by epidemiologists). This threshold can be calculated from the condition that the basic reproductive number of the phage is greater than one (Anderson \& May, 1992). The basic reproductive number, $R_{0}$, is defined as the number of secondary infections per infected cell. Each infected bacterial cell can divide and will thus give rise to a cell line which, on average, will exist for a time $1 /(k-a)$, during which this lineage will produce $L k /(k-a)$ virus particles. Each of these will cause on average $b x /(b x+m)$ new infections. The total number of secondary infections per infection is therefore

$$
R_{0}=\frac{L k}{(k-a)} \frac{b x}{(b x+m)}
$$

Because the basic reproductive number of the phage depends on the density of bacteria, the appropriate condition for $R_{0}>1$ can be expressed in a convenient form: the phage and the lytic bacteria increase in number only when $x(t)>X_{P}$, where

$$
X_{P}=\frac{m(k-a)}{b(k(L-1)+a)} \approx \frac{m(k-a)}{b k L}
$$

The concentration of bacteria may be reduced either by the host's own responses or by the action of the phage. The former occurs when the function describing the host response, $H(t)$, exceeds the basic replication rate of the bacteria, $a$. The latter occurs when the concentration of free phage, $v(t)$, exceeds a critical threshold, $V_{I}$, defined by

$$
V_{I}=\frac{a}{b}
$$

This viral inundation threshold, $V_{I}$, only expresses whether the phage can to some degree reduce the number of bacteria. Whether or not the phage will actually succeed in clearing the bacterial infection is a different matter. Under inundation therapy (passive therapy) the bacteria will be cleared if the inoculum of phage is greater than a certain threshold, $V_{C}$ say. During such clearance the role of secondary infection is negligible, and so we can approximate the behaviour of the phage by ignoring the lysis term, so that $\mathrm{d} v / \mathrm{d} t \approx-b v x-m v$. The behaviour of the uninfected bacteria is dominated by the transmission term, so that $\mathrm{d} x / \mathrm{d} t \approx-b v x$. We now consider the expression

$$
\frac{\mathrm{d} v}{\mathrm{~d} x}=\frac{\mathrm{d} v}{\mathrm{~d} t} / \frac{\mathrm{d} x}{\mathrm{~d} t}=1+\frac{m}{b x} .
$$

This has an explicit solution obtainable from integration by separation of variables. We thereby obtain a relationship between $x$ and $v$ :

$$
v(t)-v_{\phi}=x(t)-x_{\phi}+\frac{m}{b} \ln \frac{x(t)}{x_{\phi}} .
$$

To calculate the clearance threshold $V_{C}$ we apply the condition that clearance continues right up to the time when the uninfected bacteria disappear, that is $v(\tau)>V_{I}$ where $\tau$ is such that $x(\tau)=\delta$, and $\delta$ is the concentration equivalent to only one bacterial cell remaining. Substituting into the above equation and rearranging yields the condition for full clearance under passive therapy to be $v_{\phi}>V_{C}$ where

$$
V_{C} \approx V_{I}+x_{0} \mathrm{e}^{a t_{\phi}}+\frac{m}{b} \ln \left(x_{0} \mathrm{e}^{a t_{\phi}}\right)
$$

If at any point in time both the inundation threshold $V_{I}$ is exceeded by the virus concentration, and the proliferation threshold $X_{P}$ is exceeded by the bacterial concentration, then there will be active replication of the virus and explosive growth in viral numbers will ensue. In this way, the bacterial infection will rapidly be brought under control. This is the primary outcome to which biocontrol by bacteriophage therapy aspires. On the other hand, if $V_{I}$ is exceeded, 
but $X_{P}$ and $V_{C}$ are not, then there will be an overall removal of bacteria by passive infection, but there will be no apparent active replication of the virus. The concentration of virus decreases until it becomes smaller than $V_{I}$, at which point if any bacteria remain they will begin to increase once more. In this case, because there is no active replication, the phage is acting in a manner little different from antibiotic treatments, with only a temporary effect and none of the benefits of active phage replication.

If $X_{P}$ is not exceeded at the time of phage inoculation, it is still possible that, with the exponentially increasing bacterial numbers, the threshold will be surpassed at a later time. There is thus an intrinsically temporal context to the problem, imposed by the critical moment at which the bacterial density passes the proliferation threshold. We shall call this the proliferation-onset time, $T_{P}$. Whether or not active phage replication is achieved depends on two factors: whether there is still any phage present when the proliferation-onset time is reached; and/or whether the proliferation-onset time occurs prior to the time when any natural host responses manifest.

It is useful to consider the time-scale of onset of the host responses in the absence of phage. Let $T_{H}$ be the time when the host might itself gain the upper hand over the bacteria [defined as $H\left(T_{H}\right)=a$ (in the absence of phage)]. This will of course depend on the nature of the host's defence systems and the context of the infection. But, unlike $T_{P}$, this time can be measured directly by experiment, and so the precise details of the dynamics need not concern us at present: merely the existence of the host response at time $T_{H}$, without details, is enough to allow us to proceed.

Because a prerequisite of achieving "active therapy" (i.e. removal of bacteria sustained by actively replicating phage) is that the phage density exceeds the proliferation threshold before the onset of the host response (i.e. $T_{P}<T_{H}$ ), it is useful to have an estimate of the size of $T_{P}$. This is straightforward for the case when the initial inoculum of phage is less than the inundation threshold. Prior to inoculation the uninfected bacteria increase exponentially with $x(t)=x_{0} \mathrm{e}^{a t}$, and hence at the time of inoculation $x_{\phi}=x_{0} \mathrm{e}^{a t_{\phi}}$.
This allows us to calculate the proliferation onset time using the condition $x\left(T_{P}\right)=X_{P}$, to give

$$
T_{P} \approx \frac{1}{a} \ln \left(\frac{(k-a) m}{b k L x_{0}}\right)
$$

The final threshold definition relates to the requirements to prevent the phage becoming fully purged from the system prior to $T_{P}$. A certain timing and size of phage inoculum is needed for there still to be some phage present at the time when $X_{P}$ is surpassed, and thus is required for active phage replication to become possible. This threshold can be thought of either in terms of a critical inoculation size $\left(V_{F}\right)$ for a given inoculation time, or a critical inoculation time $\left(T_{F}\right)$ for a given inoculation size. Prior to $T_{P}$ the behaviour of the uninfected bacteria is dominated by the growth term, so that $\mathrm{d} x / \mathrm{d} t \approx a x$. At the same time the numbers of infected bacteria will always be very small, which means that a quasi-steadystate hypothesis can be imposed on eqn (2) (Murray, 1989), so that we approximate $y(t)$ $=b v(t) x(t) /(k-a)$ (the time taken to reach this pseudo-equilibrium state introduces a small error to the estimates given below). Substituting for $y(t)$ into $\mathrm{d} v / \mathrm{d} t$ and using eqn (4) we can write the expression

$$
\frac{\mathrm{d} v}{\mathrm{~d} x}=\frac{\mathrm{d} v}{\mathrm{~d} t} / \frac{\mathrm{d} x}{\mathrm{~d} t}=-m v\left(1-\frac{x}{X_{P}}\right) .
$$

Integration by separation of variables yields the relationship

$$
\frac{a}{m} \ln \frac{v(t)}{v_{\phi}}=\frac{\left(x(t)-x_{\phi}\right)}{X_{P}}+\ln \frac{x(t)}{x_{\phi}} .
$$

To calculate the threshold $V_{F}$ we apply the condition that some phage are still present when the proliferation onset time is reached, that is $v\left(T_{P}\right)>\varepsilon$, where $\varepsilon$ is the concentration equivalent to only one phage being present. Substituting into the above equation and writing $x_{\phi}=x_{0} \mathrm{e}^{a t_{\phi}}$ gives the condition $v_{\phi}>V_{F}$, where

$$
\begin{aligned}
V_{F} \approx & \varepsilon \exp \left(m\left(T_{P}-t_{\phi}\right)\right. \\
& \left.+\frac{m}{a}\left(\mathrm{e}^{-a\left(T_{P}-t_{\phi}\right)}-1\right)\right) .
\end{aligned}
$$


One cannot rewrite this explicitly for $T_{F}$, although a first approximation is possible when $t_{\phi} \ll T_{P}$. In this case, the condition is that $t_{\phi}>T_{F}$, where

$$
T_{F} \approx T_{P}-\frac{1}{m} \ln \frac{v_{\phi}}{\varepsilon}-\frac{1}{a}
$$

For a given initial dose size, attempts at active therapy will fail outright if treatment is made prior to the failure threshold time $T_{F}$.

\section{CATEGORIES OF BEHAVIOUR AND CATEGORIES OF OUTCOME}

By consideration of the above thresholds it is possible to delimit various categories of dynamic behaviour. The actual outcome of disease in any particular case then depends on the order of progression through those categories.

I. Passively effective. If $x_{\phi}<X_{P}$ and $v_{\phi}>V_{I}$, then both infected bacteria and phage initially decrease in number. If $v_{\phi}>V_{C}$ this passive removal leads to clearance. If not, then the bacteria decline only until the viral concentration is below the inundation threshold $\left[v(t)<V_{I}\right]$. Given sufficient time this case might progress to the delay phase (II), then to the active phase (III), and so to resolution - but only provided the host responses do not pre-emptively intervene.

IIa. Temporarily ineffective (kinetically latent). If $x_{\phi}<X_{P}$ and $v_{\phi}<V_{I}$, then the number of phage initially falls, but the uninfected bacteria increase. Eventually, $x$ will become greater than $X_{P}$, whereupon the system commences the active phase (III). The time when this happens is given in eqn (7). If the onset of host responses occurs before this time (if $T_{H}<T_{P}$ ) then the phase of active control will never be reached.

IIb. Totally ineffective. If $t_{\phi}<T_{F}$, then the number of phage declines and falls to zero before the proliferation onset time is reached, thereby precluding active replication.
III. Actively effective. If $x_{\phi}>X_{P}$ but $v_{\phi}<V_{I}$, then the phage increase rapidly with positive feedback via the transmission term. Soon $v$ will become greater than $V_{I}$ and the resolution phase (IVa) will be reached.

IVa. Resolution by active proliferation. If $x_{\phi}>X_{P}$ and $v_{\phi}>V_{I}$, then the phage increase in number, the uninfected bacteria decrease in number, and the worst of the disease will have passed.

IVb. Resolution by passive inundation. If the initial phage dosage is extremely large $\left(v_{\phi}>V_{C}\right)$ then the bacteria can be cleared by primary infection alone.

IVc. Resolution by host. If for any reason the phase IVa is not reached before time $T_{H}$, then the bacterial disease will be brought under control by the host rather than by the phage. In such a case, although the phage do not conquer the disease, they may still act to ameliorate its severity.

From these categories of behaviour only certain sequences are possible, each with different implications for the effectiveness of the phage therapy. The main categories of outcome, and the associated ordering of dynamical behaviours, are summarized in Table 2.

The difference between active and passive removal of bacteria is apparent in the experimental results of Tolkachera et al. (1981, cited in Alisky et al., 1998), who compared the use of coliProteus phage and anti-pseudomonas phage as part of treatment of dysentery in immunosuppressed leukaemia patients. Patients given anti-pseudomonas phage recovered after only one course. Patients given coli-Proteus needed 2-3 courses to effect recovery, and Proteus concentrations declined only during each course, showing renewed multiplication between courses. This clearly indicates that the coli-Proteus was only able to be passively effective, with clearance of the bacteria being achieved only as a result of repeated administration of the phage. In contrast, the behaviour of anti-pseudomonas phage is consistent with actively effective therapy. In terms of our model these different outcomes are interpreted as the types of phage having different values of the proliferation threshold $X_{P}$. The concentration of bacteria in the 
TABLE 2

Main categories of outcome

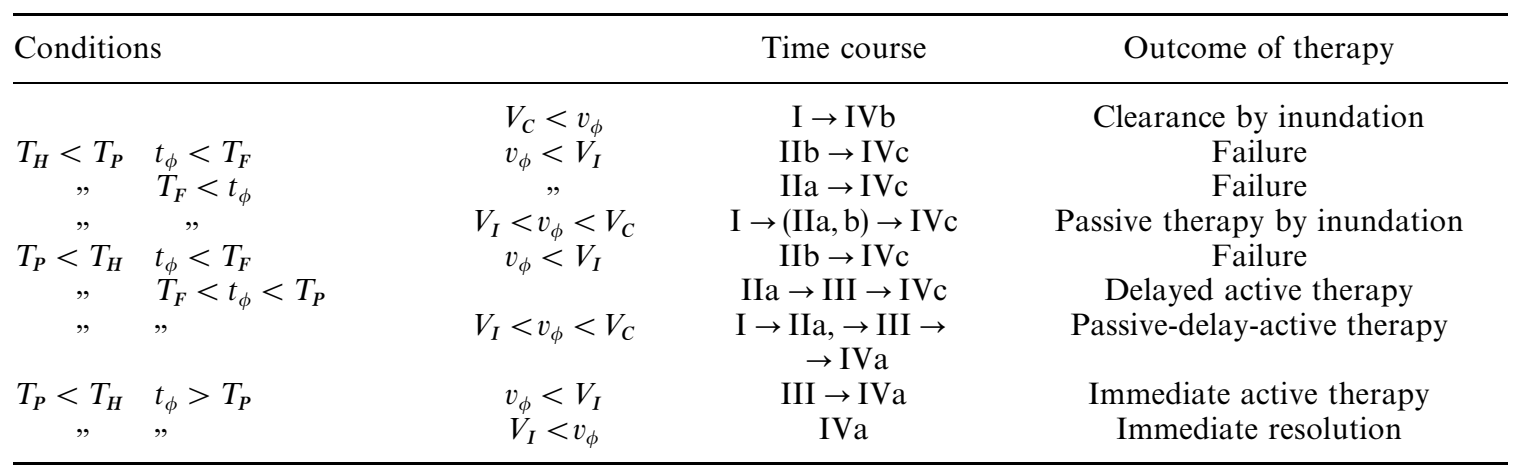

Definitions: $t_{\phi}$ time of phage inoculation; $v_{\phi}$ size of phage inoculum; $V_{I}$ inundation threshold; $V_{C}$ clearance threshold; $T_{F}$ failure threshold time; $T_{P}$ proliferation onset time; $T_{H}$ host response onset time.

patients was sufficient to support invasion and continued-secondary phage replication by antipseudomonas, but not by coli-Proteus.

Another study in which the action of the phage was only passive was reported by Berchieri et al. (1991), who used Salmonella phage to treat chicks orally infected with Salmonella typhimurium. Although mortality was reduced from 60 to $3 \%$, large numbers of phage were needed and there was no evidence of in vivo phage multiplication. This indicates that the concentration of $S$. $t y$ phimurium was less than the threshold $X_{P}$, being insufficient to support invasion by the Salmonella phage. In addition, the large number of phage required reflects the need for the phage inoculation concentration to surpass the threshold $V_{I}$. Strong in vivo active replication, implying a low and easily surpassed value of $X_{P}$, has been reported for anti-K1 phage used against E. coli infection in mice (Smith \& Huggins, 1982) and cattle (Smith et al., 1987a).

Our model predicts that actively effective therapy is dependent on the concentration of bacteria whereas passively effective therapy is dependent on the concentration of the phage. A consequence of this is that if active therapy is possible, then it may proceed even with low initial phage dosage, whereas passive therapy will not occur at all unless the initial phage dosage exceeds $V_{I}$. This is nicely illustrated in experiments by Soothill (1992) on the ability of three species of phage to control the three bacteria species Acinetobacter baumannii AC54, Pseudomonas aeruginosa 3719, and Staphylococcus aureus 6409 (all of which are implicated in infections of burns patients, and which frequently show antibiotic resistance). Treatment of $A$. baumannii with acinetobacter phage was very effective, being achieved with doses as low as one phage per $10^{6}$ bacteria, there being a $10^{5}$-fold in vivo increase in phage numbers. This is a clear case of actively effective therapy, although the data are not sufficient to distinguish between immediately active phage multiplication and delayed active multiplication. In the other two systems in vivo phage proliferation was not observed, despite being possible in vitro, suggesting these systems to have higher (unsurpassed) proliferation thresholds than in the acinetobacter system. Treatment of $P$. aeruginosa was only effective for doses of pseudomonas phage greater than $1.2 \times 10^{7}$ particles, supporting the notion that passively effective therapy requires a threshold phage concentration to be exceeded, in this case with $V_{I} \sim 1.2 \times 10^{7}$ particles. Attempts to treat $S$. aureus with staphylococcal phage failed at all dosages, implying a much higher value of $V_{I}$, and possibly also a late timing of $T_{F}$. These results illustrate how quantitative measurements of dosages required for clearance of in vitro broth culture do not provide a simple prediction of the qualitative 
nature of in vivo outcome. To extrapolate from in vitro measurements to in vivo expectations requires appreciation that kinetic behaviour is realized in a context of density-dependent thresholds.

\section{Therapeutic Implications}

The relative magnitudes of the expected time of onset of host defences $\left(T_{H}\right)$ and the projected time of initiation of active phage replication $\left(T_{P}\right)$ will be critical in determining the therapeutic outcome. The actual time of recovery cannot be precisely predicted, but in most cases will follow rapidly after whichever occurs first out of $T_{H}$ or $T_{P}$. In principle, these two time-scales could be comparable, leading to a degree of interaction, but for the present paper we restrict attention to when either one term or the other dominates.

\section{OPTIMAL TIMING}

Suppose that the biological parameters are such that $T_{H}<T_{P}$. This means that the phage never has sufficient time to bring the bacteria under control, and the only mode by which the phage may potentially contribute is via passive therapy (I). To actually do so requires a minimum dosage of $v_{\phi}>V_{I}$. Under these conditions the rule is simple: the larger and earlier the dose of phage the better. Figure 2 shows the role of $V_{I}$ and $V_{C}$ for an example in which the host response is assumed to become significant at a time of ten time units following initial infection. The calculated estimates for $V_{I}$ and $V_{C}$ are marked. We have used the maximal bacterial concentration as a simple measure of disease severity. For Fig. 2 the maximal bacterial concentration coincides with the time when the host responses first become significant (in contrast, for Fig. 3, the maximal bacterial concentration occurs when the phage gains the upper hand over the bacterial infection - cf. Fig. 1(c) and (d).

But what happens if the biological parameters are such that $T_{P}<T_{H}$, that is, if active therapy is feasible? If the initial inoculum is $v_{\phi}<V_{I}$ and $t_{\phi}<T_{F}$ then treatment is an outright failure. If, however, $t_{\phi}>T_{F}$ then we start out either in the "active" (III) or the "delayed active" (IIa) categories. In this case, the optimal time of inoculation

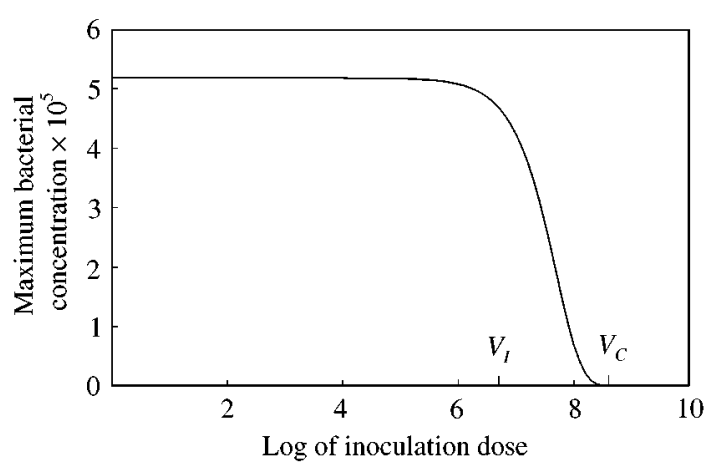

FIG. 2. Effect of inoculum size for passive therapy. The maximal bacterial concentration during infection as a function of the inoculation dose. It is assumed that the host gains control of the bacterial infection at around 10 time units $\left(T_{H} \approx 10\right)$ - thus we use the concentration of bacteria present at $t=10.0$ as a measure of disease severity. Administration of phage can ameliorate the infection by reducing the bacterial load, but this is only effective if large doses of phage are given. The predicted values of $V_{I}$ and $V_{C}$ are marked, calculated from eqns (5) and (6), respectively. Simulations were performed using various forms of host response (for example, the specific immune response form used by Levin $\&$ Bull, 1996), but it was found that in this context the details were not important. Parameters as in Fig. 1(a) and (b), with phage administered at $t=2.5$.

is actually at the boundary of these two categories, at the threshold time $t_{\phi}=T_{P}$. To inoculate earlier is to place the system in the quiescent phase during which much of the phage will be lost while waiting for the active phase to start. To inoculate later is to waste useful time during which phage could actively multiply with positive feedback. Thus, if a species of phage has the potential for active therapeutic control, then it is not true to say that earlier inoculation is necessarily better: an intermediate time is optimal.

Figure 3 gives example results of numerical solutions that illustrate both points just made. First, the severity of disease ( $y$-axis) is minimized when the time of inoculation is close to the calculated value of $T_{P}$. Second, it is possible for treatment to occur so early that no therapeutic effect at all is achieved. The failure threshold time $T_{F}$ depends on the size of the initial dose; the smaller the inoculum of phage, the later the failure threshold, and the more likely it is that no phage will be left in the system by the time active replication becomes feasible. This accounts for the sudden vertical termination of the curve towards the left-hand side. For a given initial 


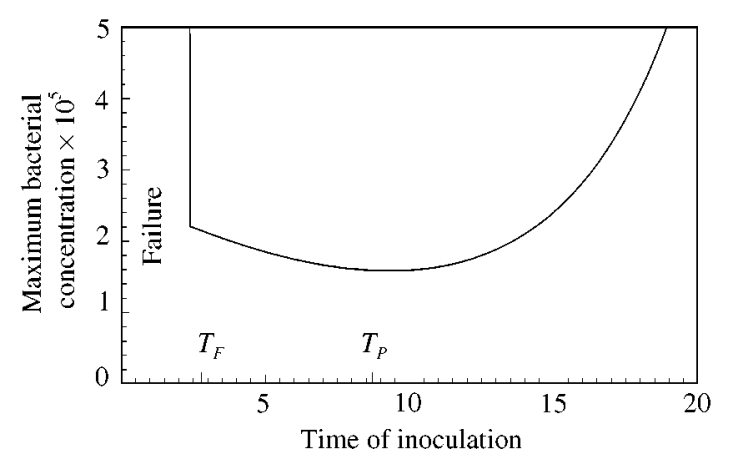

FIG. 3. Effect of inoculation time for active therapy. The maximal bacterial concentration during infection as a function of inoculation time. Here the parameter values are such that the activation onset time occurs prior to any host response, so that active therapy is possible $\left(T_{P}<T_{H}\right)$. If phage is administered too early, i.e. before the time point $T_{F}$, then treatment fails completely because the phage numbers drop to zero before the activation onset time is reached. Optimal therapeutic effects are achieved when the phage are inoculated near the proliferation onset time $T_{P}$. The predicted values of $T_{P}$ and $T_{F}$ are marked, calculated from eqns (7) and (9), respectively. Parameters as in Fig. 1 (c) and (d).

dosage it is possible to predict an approximate value of this lower failure threshold time using eqn (7).

Finally, consider what happens when there is the potential for both a period of initial passive therapy and a subsequent delayed period of active therapy (seventh entry in Table 2). An interesting point here is that, because there is initially passive removal of bacteria, the time when the proliferation threshold is passed becomes postponed. Although it is in principle possible to derive a modified form of eqn (6) to account for this type of case, it is difficult to predict the consequences for overall efficacy of therapy, and thus this situation may be best considered on a case-by-case basis. Whether or not such dualphase outcomes are ever likely to occur in practice is unclear.

Although a number of studies have presented data describing the time course of infection (Smith \& Huggins, 1982, 1983), and the importance of timing relative to feeding for oral administration has been noted (Smith et al., 1987b), there are as yet no explicit studies of the dependency of outcome upon inoculation time. The pivotal role of the proliferation onset time will only be relevant for phage that show in vivo activity, and is likely to be more readily observable for low initial bacterial doses.

\section{ANTIBIOTICS}

Few experimental studies have investigated the therapeutic potential of simultaneous treatment with phage and antibiotics. Slopek et al. (1983) found concomitant administration of antibiotics to diminish efficacy of phage therapy from 95.2 to 84.9\%. Sakandelidze (1991, cited in Alisky et al., 1998 ) found a less emphatic reduction from 86.3 to $82.5 \%$, in combination treatment of infections secondary to allergic rhinitis, dermatitis and conjunctivitis.

Our model can be adapted to predict the influence of such concomitant antibiotic inoculation, by adding an extra equation for antibiotic concentration $\alpha(t)$ of the form

$$
\frac{\mathrm{d} \alpha}{\mathrm{d} t}=-\alpha(x+y) d-\alpha n(t)
$$

and appending terms $-\alpha x c$ and $-\alpha y c$ to eqns (1) and (2), respectively. The decay term $n(t)$ may be time-dependent, according to the nature of any host responses. The consequences of using an antibiotic adjuvant depend on whether or not active viral replication occurs.

(i) Antibiotics with passive therapy. Antibiotics have a direct effect by reducing the growth rate of bacteria so that the magnitude of infection is lessened at the the point when host responses take over. There is also an indirect effect because the presence of antibiotics lowers the size of the inundation threshold, making passive removal of bacteria more likely. Both of these factors make the use of antibiotics beneficial to the operation of passive therapy.

(ii) Antibiotics with active therapy. To understand what happens when antibiotics are added on top of what would otherwise be effective active phage therapy, notice that the additional terms are qualitatively equivalent to decreasing the size of the growth parameter $a$ relative to the case without antibiotics. Hence, we can gain insight simply by examining the dependency of 


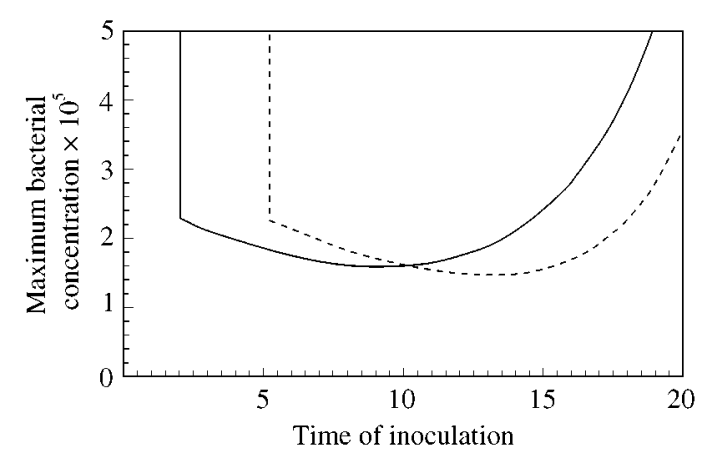

FIG. 4. Effect of using an antibiotic adjuvant. The solid line reproduces Fig. 3. The dashed line shows the result of the same simulations except with the addition of a single dose of antibiotics concomitant with phage inoculation. As predicted, the addition of antibiotics is detrimental for inoculations if made prior to the activation onset time, and beneficial if given thereafter. $\alpha(0)=1, c=10^{-6}, d=10^{-6}$, $n=1$; other parameters as in Fig. 3 .

the critical thresholds on the size of $a: \mathrm{d} X_{P} / \mathrm{d} a=-m / b k L$ and $\mathrm{d} T_{P} / \mathrm{d} a=-1 / a$ $\left(T_{P}+1 /(k-a)\right)$. Hence, adding antibiotics increases both the proliferation threshold and the proliferation onset time. But, because the optimal inoculation time is at $t_{\phi}=T_{P}$, the effect of the addition of antibiotics depends on whether or not the consequent increase in $T_{P}$ brings it closer to $t_{\phi}$. If $t_{\phi}>T_{P}$ then increasing $T_{P}$ will be beneficial, in which case the presence of antibiotics will enhance the effect of phage therapy. But if $t_{\phi}<T_{P}$ then increasing $T_{P}$ lengthens the duration of the quiescent phase, and thus reduces the period of active therapeutic value, this being detrimental to the efficacy of phage therapy.

Equation (7) points to another, possibly more important consequence of increasing $T_{P}$. When using an antibiotic adjuvant, the failure threshold time $T_{F}$ is pushed back in time (to $T_{F}^{*}$, say), making outright failure of therapy more likely. Figure 4 illustrates both of these phenomena.

\section{Discussion}

Many ideas have been offered as to why studies of phage therapy are so prone to failure. We suggest that an important factor has been a lack of awareness of density-dependent phenomena that are intrinsic to self-replicating agents, but that do not occur with standard pharmaceuticals. Although failure of therapy is often attributed to low activity in vivo compared with in vitro, this is not of itself a reason: one must understand why there should be such a disparity between in vivo and in vitro.

In kinetic terms there are four basic issues important in determining the nature of the outcome of phage therapy. (i) Is the phage able to cause a net reduction in bacterial numbers simply by inundation ("passive therapy")? This requires the concentration of phage to exceed the inundation threshold $\left(V_{I}\right)$. (ii) Is the phage able to bring about complete clearance by inundation alone? This requires the concentration of phage to exceed a higher threshold, the clearance threshold $\left(V_{C}\right)$. (iii) Is the phage able to persist long enough to commence active replication ("active therapy")? This depends both on the bacterial numbers exceeding a specific level which we call the proliferation threshold $\left(X_{P}\right)$, and on the timing of phage inoculation being late enough [later than $T_{F}\left(v_{\phi}\right)$ for given initial dose $v_{\phi}$ ]. (iv) Can active replication bring the bacterial infection to crisis before the onset of any natural host response? This requires the proliferation onset time to be less than the time-scale of natural host responses $\left(T_{P}<T_{H}\right)$.

We have used the phrase "passively effective" to describe cases when most bacteria are removed by lysis following primary infection by the inoculated phage, whereas by "actively effective" we mean that secondary infection by lysis-released phage is the dominant cause of bacterial removal. The difference between actively and passively effective removal is a dichotomy that pervades all forms of biocontrol. For instance, application of baculoviruses as insecticides can either be inundative, with the objective of a rapid kill time, or low level, with the aim of taking advantage of the natural virus replication. The secondary infection resulting from virus replication can lead to a higher insect mortality overall, but acts only over a longer time-scale (Fuxa, 1987; Hauxwell, 1999). To know whether active therapy may occur in any particular system requires consideration of relative timings. In the bacteria-bacteriophage system one must compare four critical time points, 
$t_{\phi}$ (time of inoculation by phage), $T_{F}$ (time after which phage must be added in order to ensure subsequent active replication), $T_{P}$ (time after which active biocontrol can occur, assuming absence of host response), and $T_{H}$ (time at which the host would control the bacteria, assuming absence of phage).

Although passive biocontrol can be effective, it will usually require large and repeated dosages for success to be ensured. In contrast, any biocontrol agent with in vivo activity need only be given as one dose, and typically that dose need only be small. We argue that to maximize this benefit, the phage inoculation should be administered as close as possible to the proliferation threshold, $T_{P}$. This prediction from our model could easily be tested experimentally. It is worth noting that the time of $T_{P}$ will be later, and hence easier to detect, for smaller initial doses of bacteria. Most laboratory studies, based on $\mathrm{LD}_{50}$ and/or $\mathrm{PD}_{50}$ measures, use large bacterial doses that may obscure this effect, and that do not accurately mimic the probably small inoculum size in natural systems. All of the parameters defining $T_{P}$ [eqn (7)] are in principle independently measurable, and so it may even be feasible to predict values of $T_{P}$ for specific study systems. Because the parameters are biologically meaningful, most of them should be measurable in vitro. It should also be practicable to test our predictions concerning $T_{F}$ [eqn (9)], and $V_{I}$ [eqn (5)], and concerning the consequences of simultaneous administration of antibiotics.

In this report, we have used the simplifying assumption that if any host response occurs, it will be relatively rapid and complete. Not all real situations will be of such a simple form, having perhaps only slow or partial clearance of the bacteria by the host. In such cases, the functions $H(t)$ and $h(t)$ which describe the host responses would have to be modelled accordingly, perhaps with their own dynamic equations (such as the dynamic immune response in Levin \& Bull, 1996). Although such cases will need more complex mathematics, the thresholds and other phenomena we have described will nonetheless remain relevant, albeit with formulae modified to account for the details of each particular scenario. Modelling the immune dynamics of specific systems will be an important objective of future studies.
Most models of phage-bacteria interactions have been aimed at addressing co-evolutionary questions. To date, the only serious attempt to model the dynamics of phage therapy as a population biology phenomenon was that by Levin \& Bull (1996), whose model was designed to reflect data from the Smith \& Huggins (1982) study of phage used against $E$. coli infection in mice. The mathematical formulation presented by Levin and Bull did not include a term for loss of phage, and for this reason neither the proliferation threshold, $X_{P}$ nor any of the consequent phenomena, would have been apparent. The fact that a significant proportion of laboratory studies exhibit only passively effective therapy clearly indicates loss of phage to be an important factor in phage-bacteria population dynamics, and underlines the necessity of including a phage loss term in our model. Merril et al. (1996) described a study specifically aimed at reducing the magnitude of phage loss, by selecting for long-circulating strains of $E$. coli phage $\lambda$ and of $S$. typhimurium phage P22, able to avoid entrapment by the reticuloendothelium system. In our model, this is equivalent to decreasing the size of $m$, the parameter for rate of phage loss. This consequently reduces the size of the proliferation onset time, the failure threshold time, and the clearance threshold. Our formulae make explicit the mechanisms by which these effects occur. Long-circulating strains will also aid potential use of phage in a prophylactic rather than merely curative capacity (cf. Smith et al., 1987a, b).

Our model is able to mimic a range of observed behaviours without recourse to inclusion of the details of the pathophysiology. This argues not only for the robustness and generality of our model, but also illustrates that many of the apparently paradoxical aspects of phage therapy are not of genetic or molecular origin, but arise from nonlinear density-dependent phenomena. A natural consequence of the density-dependent nature of the phage-bacteria interaction is that the timing of events is important. For most diseases it is important to administer treatment as early as possible, yet we have shown this not to be the case for phage therapy. If one wishes to benefit from active phage replication then early treatment can be detrimental. Moreover, using parallel administration of phage and antibiotics 
is a practice one should be wary of - as we have shown, the antibiotics can often act against the interests of the phage. Both of these counterintuitive, yet clinically important issues, only make sense when viewed as density-dependent phenomena constrained by critical time points. Even if variation amongst individual patients cannot be resolved at a clinical level, a quantitative understanding of phage-bacteria kinetics is still of significant benefit (Payne \& Jansen, 2000), for it informs us which aspects of phage biology might best be engineered so as to enhance the prospects of phage therapy.

The reviews of Barrow \& Soothill (1997) and Alisky et al. (1998) both conclude that bacteriophage show increasing promise as antimicrobial agents, warranting greater investment in their investigation and development. The replication of phage, their narrow host range, and their potential for directed evolution, gives phage therapy a number of unique advantages over antibiotics. To profit from the putative benefits of phage therapy will not only require intimate knowledge of the pathogenesis of infection, as discussed by Barrow \& Soothill (1997), but will also depend upon interpreting that knowledge within the density-dependent framework that we have set forth above.

This work was funded by the NERC (RJHP) and by The Wellcome Trust grant no. 051319 (VAAJ). We thank Bob May and Hester Korthals Altes for advice.

\section{REFERENCES}

Alisky, J., ICZKOWSKI, K., RAPOPORT, A. \& TrOITSKY, N. (1998). Bacteriophage show promise as antimicrobial agents. J. Inf. 36, 5-15.

ANDERSON, R. M. \& MAY, R. M. (1992). Infectious Diseases of Humans. Oxford: Oxford University Press.

Barrow, P. A. \& Soothill, J. S. (1997). Bacteriophage therapy and prophylaxis: rediscovery and renewed assessment of potential. Trends Microbiol. 5, 268-271.

Berchieri, A., Lovell, M. A. \& BArrow, P. A. (1991). The activity in the chicken alimentary tract of bacteriophages lytic for salmonella typhimurium. Res. Microbiol. 142, 541-549.

BERKOWITZ, F. E. (1995). Antibiotic resistance in bacteria. South Med. J. 88, 797-804.

D'HERELle, F. (1917). Sur un microbe invisible antagoniste des bacilles dysentériques. Comptes Rendus Acad. Sci. 165, 373-375.
FUXA, J. R. (1987). Ecological considerations for the use of entomopathogens in IPM. Ann. Rev. Entomol. 32, 225-251.

HAUXWELL, I. C. (1999). Evaluation of potential baculovirus insecticides: studies of the infection process and host susceptibility. Ph.D. Thesis, London University.

LeVIN, B. R. \& BulL, J. J. (1996). Phage therapy revisited: the population biology of a bacterial infection and its treatment with bacteriophage and antibiotics. Am. Nat. 147, 881-898.

Merril, C. R., Biswai, B., Carlton, R., Jensen, N. C., Creed, G. J., Zullo, S. \& AdhiYA, S. (1996). Long-circulating bacteriophage as antibacterial agents. Proc. Natl Acad. Sci. U.S.A. 93, 3188-3192.

Murray, J. D. (1989). Mathematical Biology. Berlin: Springer-Verlag.

Payne, R. J. H. \& Jansen, V. A. A. (2000). Phage therapy: the peculiar kinetics of self-replicating pharmaceuticals. Clin. Pharm. Therap. 68, 225-230.

SAKANDELIDZE, V. M. (1991). Kompleksnoe primenie spetsificheskikh fagov i antibiotikov pri razlichnykh infektsionnykh allergozakh. [The combined use of specific phages and antibiotics in different infectious allergoses]. Vrachebno Delo 3, 60-63 (in Russian).

Schrag, S. J. \& Mittler, J. E. (1996). Host-parasite coexistence: the role of spatial refuges in stabilizing bacteria-phage interactions. Am. Nat. 148, 348-377.

SlopeK, S., Durlakowa, I., Weber-DabrowsKa, B., KucharewiCZ-KrukowskA, A., DABrowski, M. \& BISIKIEWICZ, R. (1983). Results of bacteriophage treatment of supparative bacterial infections I. General evaluation of the results. Arch. Immunol. Therap. Exp. 31, 267-291.

Smith, H. W. \& Huggins, M. B. (1982). Successful treatment of experimental Escherichia coli infections in mice using phage: its general superiority over antibiotics J. Gen. Microbiol. 128, 307-318.

Smith, H. W. \& Huggins, M. B. (1983). Effectiveness of phages treating experimental Escherichia coli diarrhoea in calves, piglets and lambs. J. Gen. Microbiol. 129, 2659-2675.

Smith, H. W., Huggins, M. B. \& Shaw, K. M. (1987a). The control of experimental Escherichia coli diarrhoea in calves by means of bacteriophages. J. Gen. Microbiol. 133, 1111-1126.

Smith, H. W., Huggins, M. B. \& Shaw, K. M. (1987b). Factors influencing the survival and multiplication of bacteriophages in calves and in their environment. J. Gen. Microbiol. 133, 1127-1135.

SoOTHILL, J. S. (1992). Treatment of experimental infection of mice with bacteriophages. J. Med. Microbiol. 37, 258-261.

Tenover, F. C. \& Hughes, J. M. (1996). The challenges of emerging infectious diseases: development and spread of multiply-resistant bacterial pathogens. JAMA 275, 300-304.

Tolkachera, T. Y., Abakumova, E. M., Martynova, V. A. \& Golosova, T. V. (1981). Korrektsiia disbakterioza kishechnika biologicheskimi preparatami u bol'nykh ostrymi leiozami. [Correction of intestinal dysbacteriosis with biological preparations in acute leukemia]. Problemy Germatologii i Perelivaniia Krovi 26, 29-33 (in Russian).

TwOrT, F. W. (1915). An investigation on the nature of the ultra-microscopic viruses. Lancet 2, 1241. 